\title{
Probability of default estimation, with a reject option
}

\author{
Lize Coenen \\ Dep. of Business techn. \& Operations \\ Vrije Universiteit Brussel \\ Brussels, Belgium \\ Lize.Coenen@vub.be
}

\author{
Ahmed K. A. Abdullah \\ Dep. of Business techn. \& Operations \\ Vrije Universiteit Brussel \\ Brussels, Belgium \\ Ahmed.Kareem.A.Abdullah@vub.be
}

\author{
Tias Guns \\ Dep. of Business techn. \& Operations \\ Vrije Universiteit Brussel \\ Brussels, Belgium \\ Tias.Guns@vub.be
}

\begin{abstract}
Many companies, such as credit granting companies, have to decide on granting or denying customer or invoice loans on a daily basis. Increasingly, machine learning is used to learn probability-of-default models from previously granted cases and, thus, whether the outcome was positive or negative for the company, i.e. whether the client paid back or defaulted. However, as the outcome can only be observed for the granted cases, the data inherently has sample selection bias and caution should be taken when applying the probability-of-default model to the full through-the-door population. In reject inference, this problem is studied with respect to whether using the unlabeled rejected instances can help improve a classifier that is only trained on granted instances, e.g. using semi-supervised learning. In contrast, we investigate under what circumstances a model trained on the granted instances, with known outcome, can be used on all possible instances. For this, we believe a model should indicate when it cannot reliably predict the outcome. That is, it should refrain from making predictions on instances unlike those on which it was trained. If not, the credit granting company would expose itself to great risk, and experts could lose their trust in the predictions. We discuss similarities and differences of this problem compared to novelty detection, classification with a reject option and reject inference. We compare a number of methods that combine novelty detection with classification, with decent results even for two-stage methods and especially when using data of existing instances with unknown outcome.
\end{abstract}

Index Terms-Reject Inference, Predictive Confidence, Machine Learning

\section{INTRODUCTION}

Credit granting companies, among others, need to decide on a regular basis whether to grant or deny loans. When granting a loan, they face the risk that the customer will not (re)pay the amount owed and hence will default. The company can observe whether the customer did or did not pay back, and learn to estimate the risk that specific customer requests entail. Indeed, accurate credit risk modelling is at the core of this business problem. Increasingly, machine learning models are used to estimate this risk. More specifically, probabilistic classifiers are used where the confidence in predicting 'default' is interpreted as the probability-of-default (PD) of the customer [1].

However, only for a portion of the customers one can observe whether they default or not. These are the instances for which the outcome is known. Other customers are denied credit because they are considered as potential defaulters or because of other business reasons. Their outcome is hence unknown. For those customers, there is no feedback on whether the right decision was made or not, e.g. whether counterfactually the customer would have defaulted or not if the loan had been granted. Standard machine learning techniques can only learn from instances with an observed outcome. This is challenging. On the one hand, there are certainly some types of customers that no expert would accept (bad credit history, unrealistic amounts, etc.) yet we do not want to grant such cases credit just to observe that they indeed default and have the system learn from that. On the other hand, new types of invoices, from new countries or markets, can turn up and client demographics change throughout time. For these cases, there is the potential to learn from similar earlier cases and make probability-of-default predictions on those, hence identifying new business opportunities.

More generally, the question we investigate is whether one could use a model trained on the granted cases, and get reliable predictions for any instance. The general answer is a clear no, as there is sample selection bias in the granted cases and hence new instances may belong to parts of the instance space that the model has never observed, and may even break the i.i.d. assumption of being identically distributed to the granted training instances.

For example, Fig. 1 shows a 2D visualization of 2 years of real-life invoice factoring data, both granted (with good or bad outcome) and rejected cases. The original data has 119 dimensions and in this 2D PCA projection the good and bad instances are difficult to separate; however it is clear that a fair number of unknown instances overlap with observed instances, but that many more do not. For these, we would not want a classifier to pretend it can estimate the probability-of-default.

The main challenge in the probability-of-default setting is that every instance inherently belongs to one of the two classes: either a customer will default or it will not. However, for the unknown instances, we cannot evaluate the accuracy of the predictive model. Out-of-distribution and novelty detection are two related tasks, focused specifically on detecting outof-distribution and novel instances. Often distance-based [31] or density-based [2] methods are used. The task we consider requires joint novelty detection and probability-of-default estimation. 


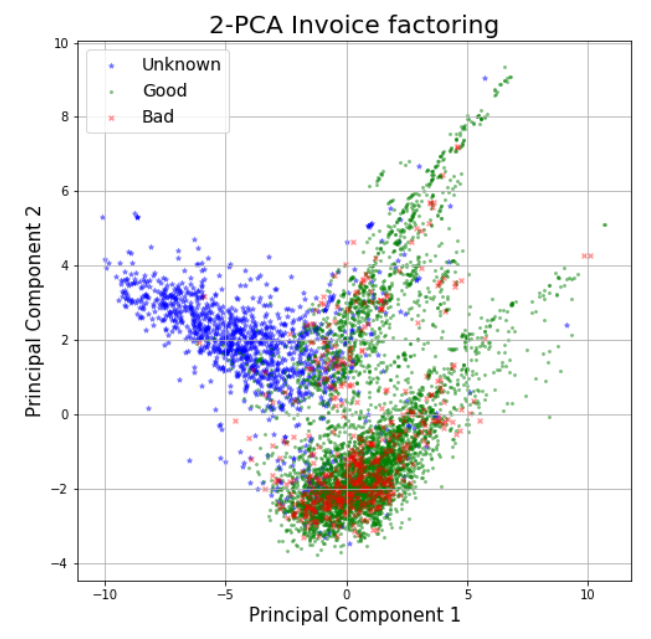

Fig. 1: Visualization of first two principal components of reallife factoring data - the full dataset has 119 dimensions.

This problem is related to 'classification with a reject option', also known as 'selective classification' and 'abstaining classifiers'. However, the motivation behind such methods often stems from the fact that the labels on the data cannot be trusted: noise in the labeling [33] or ambiguous labels [7]. Such methods often use the class probability values to estimate whether to predict a class or not. This type of reject is called ambiguity reject [4]. In a sense, these methods 'know' what to predict but their predictions point to several classes, e.g. in case of instances close to the decision boundary. In binary classification and probability-of-default estimation, the class probability already expresses this ambiguity.

Instead, we are interested in rejecting instances that are unfamiliar to the learning method and hence instances about which it makes arbitrary (confident or unconfident) predictions. These instances might be located far from the decision boundary, but are located away from known instances as well. This is called distance reject [6] and to avoid confusion with distance-based methods we will refer to it as novelty reject.

The literature on reject inference has been investigating the potential of doing inference on unknown (denied) instances for many years. The initial hope was that inference on unknown instances could be used to boost test accuracy on known instances (similar to semi-supervised learning), but several negative results have been reported [9] [3]. Instead, our goal is to discard novel denied/unknown instances while only providing predictions for samples that are similar to the instances the method was trained on.

In this paper, we hence investigate the potential of a range of techniques that are related to classification with reject option and novelty detection. We investigate their assumptions, applicability to probability-of-default estimation and technical means. We further compare the different methods on seven datasets, of which one artificial and two from the credit scoring domain.

Our main contributions are the following:

- We enrich the common practice of estimating the probability-of-default for decision support in credit risk modelling by including the option to reject unfamiliar instances;

- We compare related methods in novelty detection, classification with a reject option and selective classification from a methodological point of view;

- We empirically investigate the feasibility of the approaches on seven datasets of which two credit scoring datasets, one public and one private;

- We further investigate the potential of rejecting unknowns for classical semi-supervised reject inference

\section{RELATED WORK}

We review related work in novelty detection, classification with a reject option and reject inference.

a) Novelty detection: We speak of novelty detection instead of outlier detection, because in outlier detection the assumption is that there are outliers in the known data; for example due to noise. In contrast, we can assume that all known instances have their true labels and instead want to detect instances that are different, that are novel. Traditional techniques for novelty detection that are used in this work are one-class SVMs [26] and isolation forests [16]. Recent work has mainly targeted out-of-distribution detection in deep neural networks (DNNs) [5] [18] [19]. Neural networks are known to fail silently when faced with test data from a different distribution than the training data, i.e. they can be overly confident about incorrect predictions. Hendrycks and Gimpel illustrate this in [11] by showing how DNNs trained on MNIST images can often produce highly confident predictions (with a probability of $91 \%$ ) on random noise images. Although they acknowledge that separate prediction probabilities from a softmax distribution are not directly suitable for confidence estimation, they argue that such probabilities can still serve as a baseline for evaluation of out-of-distribution detection methods by showing that the prediction probabilities of incorrectly classified and out-of-distribution instances tend to be lower than the prediction probabilities for correctly classified instances. They demonstrate the effectiveness of such a baseline but show that there is room for future research.

Liang e.a. [15] developed an Out-of-Distribution detector for Neural networks (ODIN) that does not require retraining of the neural network, uses temperature scaling in the softmax function and adds small perturbations to the input. ODIN improves on the baseline. In [25], Schultheiss e.a. propose to use convolutional neural networks for novelty detection by exploiting the difference in class-specific activation patterns between instances of known and unknown classes. While [15] and [25] assume a pre-trained network or at least preexisting knowledge about the 'known' population to identify novel samples, Lee e.a. [13] use an adverserial network to generate 'boundary' samples in between in-distribution and out-of-distribution samples, jointly train a model to detect 
out-of-distribution samples and show its effectiveness. We do not limit ourselves to pre-trained networks, but do assume unknown (test) instances are available.

b) Classification with a reject option: Standard classification with a reject option is focussed on the ambiguity reject. Thulasidasan e.a. [34] propose a deep abstaining neural network that trains on a novel loss function that enables robust learning by learning representations for noisy samples and samples from unknown classes. Recently, a bounded abstaining classifier with two constraints of reject rates (BA2Cs) was proposed by Guan e.a. [8] to facilitate class-specific reject rates and imbalanced datasets. In [7], Geifman and El-Yaniv use deep neural networks for classification with a reject option, i.e. selective classification. For a given trained network and a given desired risk bound, they construct a selective classifier that trades off coverage for risk. As this technique uses the most straightforward way to rate confidence, i.e. the softmax response, we will use it in our experiments as a baseline method for classification with ambiguity reject.

In contrast, Tax and Duin [32] investigate the notion of classification with a reject option under distance reject and, more broadly, novelty detection. They consider a multi-class setting and propose a heuristic for combining one-class models.

Perhaps most related to our setting is Background Check [22] and we will therefore use it in our experiments. It concerns a technique that does not depend on a specific type of base classifier. It allows classifiers to assess the difference between unlabeled test data and the data they were trained on by making an explicit difference between foreground and background distribution.

c) Reject Inference: By investigating what added value the inclusion of rejected instances can provide to a credit risk model, our approach is related to the process of reject inference, which tries to make a credit risk model's training data representative for the through-the-door population by including data of rejected applicants and inferring their outcome.

Over the years, various reject inference methods have been developed. Common straight-forward approaches use the known good/bad model or probability of acceptance to make inferences about the rejected instances and their performance is therefore heavily dependent on the quality of the original scoring model [28]. Another trend concerns the usage of statistical correction techniques for sample selection bias, including Heckman's well-known bivariate two-stage model [10], which models the accept/reject decision as well as the $\mathrm{good} / \mathrm{bad}$ outcome. This model however requires a strong normality assumption of the error terms and exclusion restrictions on the variables and is shown to be inefficient for cases with strong selection bias [23].

Recently, reject inference has been approached as the machine learning task of semi-supervised learning. In [17], Maldonado and Paredes apply a semi-supervised approach to reject inference by self-training an SVM classifier using the probabilistic output after Platt scaling as a confidence measure. In this regard, Tian e.a. [35] proposed an approach based on the state-of-the-art kernel-free fuzzy quadratic surface SVM model, preceded by outlier detection, to learn accepted applicants who defaulted to reduce their negative influence during training. Xia e.a. [37] took it a step further and inferred the status of rejected applicants by using isolation forest, followed by a LightGBM model trained on all (inferred) labels as part of a novel model named OD-LightGBM. While similar in spirit to ours, it uses outlier detection to label all unknowns that are then used for training, while our aim to use outlier detection is to reject novel instances.

Kozodoi e.a. [12] proposed a self-learning framework for reject-inference. Their framework starts out filtering and dropping rejected applicants that are either too similar or not similar enough to the distribution of accepted cases using isolation forest as well. During self-learning the method then iteratively adds the most confident predictions for unknowns to the training set using class probability as a confidence measure. We will introduce a similar self-learning method in Section VI which skips the preliminary filtering.

All in all, the methods described above perform with varying degrees of success. Both Hand and Henley [9] and Chen and Astebro [3] conclude that the performance of reject inference methods is not reliable. This makes the question of whether we should try to infer the labels of all unknown instances all the more pertinent.

\section{PROBLEM SETTING}

In a binary classification setting, our goal is to reject instances that are relatively 'unknown' to the classifier, and only provide a class probability estimate for instances that are sufficiently familiar (non-novel) to it, that is, for which it can reliably make a probability estimate.

We assume the availability of a labeled dataset $\left\{\left(X_{i}^{k}, y_{i}\right)\right\}$ with binary labels $y_{i} \in\{+,-\}$, this datasets will be called the known instances; as well as an unlabeled dataset $\left\{\left(X_{i}^{u}\right)\right\}$ of unknown instances: instances whose label is unobserved (e.g. 'rejected' cases for credit granting companies) and for which we hence do not know the outcome. Note that each instance can only have one of the two outcomes: either it would have defaulted or not, but this information is unknown due to not having started a relationship with the client. Furthermore we can NOT assume that these instances are identically distributed with respect to the known instances, they are defined over the same feature space but may be from entirely different parts of the feature space.

The intermediate goal is to build a classifier that outputs two values:

$$
(s, p)=f\left(X_{i}\right)
$$

where $s$ is the novelty score that indicates to what extent the instance is 'novel'; that is, unlike the known instances from the training set. Note that this score $s$ may be a probability, e.g. the probability that it does not belong to the distribution of the known data: $P\left(X_{i} \notin \Pi\left(X^{k}\right)\right)$, or just a free-ranging value such as a distance. The second value $p$ is the probability of being a case that will default, assuming it belongs to the same distribution as the known data, e.g. $P\left(y_{i}=+\mid X_{i}, X_{i} \in\right.$ 
$\left.\Pi\left(X^{k}\right)\right)$. This is the traditional 'probability of default' of an instance $X_{i}$.

We will write $s\left(X_{i}\right)$ to refer to the first component of $(s, p)=f\left(X_{i}\right)$ and $p\left(X_{i}\right)$ to refer to the second component. The endgoal is a probability-of-default estimator with a reject option to decide whether an instance should be rejected or not. That is, part of the training process is to decide on a threshold $\theta$ on the novelty score, such that either -1 or the probability-of-default is returned:

$$
p^{\prime}\left(X_{i}\right)= \begin{cases}-1 & \text { if } s\left(X_{i}\right) \geq \theta \\ p\left(X_{i}\right) & \text { if } s\left(X_{i}\right)<\theta\end{cases}
$$

Likewise, we can turn it into a binary classifier with reject option by determining a class probability threshold $\gamma$ that determines, on top of the above, whether the classifier predicts 'positive' or 'negative':

$$
y^{\prime}\left(X_{i}\right)= \begin{cases}\text { reject } & \text { if } s\left(X_{i}\right) \geq \theta \\ + & \text { if } s\left(X_{i}\right)<\theta \text { and } p\left(X_{i}\right) \geq \gamma \\ - & \text { if } s\left(X_{i}\right)<\theta \text { and } p\left(X_{i}\right)<\gamma\end{cases}
$$

where $\gamma$ may be set to 0.5 or empirically estimated using costsensitive thresholding methods [27].

\section{Evaluating PD With A REJECT OPTION}

We assume the presence of unknown, novel cases $X^{u}$ both in the training and in the test data. This makes evaluation inherently difficult as there is no 'ground truth' available for the unknown instances. In fact, the mere presence of ground truth would invalidate the concept of having 'unknowns'.

Furthermore, we cannot simply assume that the unknowns should get a novelty reject, as some instances may be from the same distribution as the known instances and hence deserve a prediction. In credit scoring, the latter ones correspond to unexplored instances whose prediction would have had a high probability of matching the outcome if the credit granting company had accepted it. In fact, instances that have been accepted before but turned out to typically default can be expected to be refused in the future and hence end up in the 'unknown' category over time. These instances should not get a novelty reject. Hence, evaluation cannot simply ignore unknowns nor assume all unknowns should be rejected.

Prior work in classification with a reject option assumes when evaluating that either (artificial) novel instances are known and should be rejected [32], or do not assume 'unknown' instances and evaluate purely on the known instances that are not rejected by the classifier [7]. Similarly in reject inference (which does assume a set of 'unknown' instances) either assumptions are made on the proportion of unknowns that should be 'accepted' or evaluation is again only on the accuracy of the known instances [17].

In binary classification, the confusion matrix is a common tool to summarize classifier performance by showing the absolute frequencies of predictions in rows, and true labels in columns; and many measures such as precision, recall and accuracy can be derived from it [29]. In our case, we have
TABLE I: Confusion matrix with additional 'unknown' in-

\begin{tabular}{|c|c|c|c|c|c|}
\hline & \multicolumn{3}{|c|}{ true label } & \\
\hline & & + & - & unknown & \\
\hline \multirow{3}{*}{ 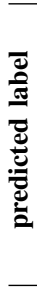 } & + & $\begin{array}{c}\text { true } \\
\text { positive } \\
\text { TP }\end{array}$ & $\begin{array}{c}\text { false } \\
\text { positive } \\
\text { FP }\end{array}$ & $\begin{array}{c}\text { unknown } \\
\text { positive } \\
\text { UP }\end{array}$ & $\begin{array}{c}\text { positive } \\
\text { predictions }\end{array}$ \\
\hline & - & $\begin{array}{c}\text { false } \\
\text { negative } \\
\text { FN }\end{array}$ & $\begin{array}{c}\text { true } \\
\text { negative } \\
\text { TN }\end{array}$ & $\begin{array}{c}\text { unknown } \\
\text { negative } \\
\mathbf{U N}\end{array}$ & $\begin{array}{c}\text { negative } \\
\text { predictions }\end{array}$ \\
\hline & reject & $\begin{array}{c}\text { positive } \\
\text { reject } \\
\text { PR }\end{array}$ & $\begin{array}{c}\text { negative } \\
\text { reject } \\
\text { NR }\end{array}$ & $\begin{array}{c}\text { unknown } \\
\text { reject } \\
\mathbf{U R}\end{array}$ & $\begin{array}{c}\text { reject } \\
\text { predictions }\end{array}$ \\
\hline & & positives & negatives & unknowns & \\
\hline
\end{tabular}
stances and 'reject' predictions.

a three-by-three confusion matrix (see Table I), reflecting the possibility that an instance can also have an 'unknown' label, as well as that the classifier can choose the 'reject' option.

Two metrics from selective classification [7] are relevant here, namely the tension between coverage, i.e. the amount of known instances which the method does not reject, and the risk within this coverage, expressed as $0 / 1$ loss. Both concepts are oppositely related to two other metrics, i.e. rejection rate and accuracy [20]:

$$
\begin{gathered}
\text { rejection rate }=\frac{\mathbf{P R}+\mathbf{N R}}{\text { positives }+ \text { negatives }} \\
\text { accuracy }=\frac{\mathbf{T P}+\mathbf{T N}}{\mathbf{T P}+\mathbf{F P}+\mathbf{F N}+\mathbf{T N}}
\end{gathered}
$$

Both only consider known cases and can be visualized on an accuracy-rejection curve (ARC) [20], which shows the accuracy (y-axis) obtained for increasing rejection rates ( $\mathrm{x}$ axis). ARCs are commonly used to evaluate classifiers that can reject instances with low classification confidence, e.g. ambiguity reject, but are also suitable for evaluating the impact of a reject option in general.

The above measures do not include the unknowns. Based on our extended confusion matrix, we motivate a number of metrics that do include the unknowns:

- Unknown reject recall is the proportion of unknowns that are rejected by the classifier:

$$
\text { unknown reject recall }=\frac{\mathbf{U R}}{\text { unknowns }}
$$

Similarly we can define positive reject recall and negative reject recall.

- Unknown-is-positive accuracy assumes that all unknown cases should be labeled positive (defaulting). While this may be true for many unknown cases, it is not true in general. It can still provide information on how classifications behave under this assumption:

$$
\text { unknown-is-pos accuracy }=\frac{\mathbf{T P}+\mathbf{U P}+\mathbf{T N}}{\text { pos. }+ \text { neg. predictions }}
$$

- Reject-is-positive accuracy takes the simplified assumption that all rejected instances will be treated as 'defaulting' and hence 'positive' by a decision maker that uses 
these predictions; hence positive/negative rejects can be treated as true/false positives:

$$
\text { reject-is-pos accuracy }=\frac{\mathbf{T P}+\mathbf{P R}+\mathbf{T N}}{\text { positives }+ \text { negatives }}
$$

\section{Methods}

We now describe four methods for constructing a probability-of-default estimator with a reject option, which implement (1). The first two methods are 'two-stage' methods, where the first stage independently determines whether an instance is novel or not, and the second stage is a standard probabilistic classifier whose output is only shown in case the instance is not novel. The other two methods have a more tightly integrated approach to the novelty detection and probabilistic classification.

\section{A. Two-stage with unsupervised novelty detection}

We propose as baseline the use of an unsupervised novelty detector, followed by a probability-of-default estimator. For the first stage, we train an unsupervised learning method on the known instances but without using the labels: $\left\{\left(X_{i}^{k}\right)\right\}$. This learns a novelty scoring function $s_{\text {unsup }}(X)$ and a threshold $\theta_{\text {unsup }}$ that determines whether the instance is novel or not.

The resulting probability-of-default estimator with a reject option is the following:

$p_{\text {unsup_pd }}(X)= \begin{cases}-1 & \text { if } s_{\text {unsup }}(X) \geq \theta_{\text {unsup }} \\ P_{2}^{p d}(y=+\mid X) & \text { if } s_{\text {unsup }}(X)<\theta_{\text {unsup }}\end{cases}$

Different unsupervised novelty detection methods can be used, such as isolation forests [16] and one-class SVMs [26]. (Kernel) density estimation and nearest-neighbor distances can also be used to determine a novelty score $s()$ but require an additional mechanism to determine the threshold $\theta$.

\section{B. Two-stage with supervised novelty detection}

This two-stage approach can only be applied in case a set of 'unknown' data $\left\{\left(X_{i}^{u}\right)\right\}$ is available. The method makes the simplifying assumption that most 'unknown' instances are novel, and different from the known instances. Hence, a method that can successfully classify the 'unknowns' from the 'knowns' can act as a novelty detector. In other words in the first stage, we use standard supervised learning of a probabilistic classifier $P_{1}^{\text {sup }}$ that was trained on a dataset where all 'unknown' instances are labeled positive and all 'known' instances negative: $\left\{\left(X^{u},+\right)\right\} \cup\left\{\left(X^{k},-\right)\right\}$.

The second stage is a standard probabilistic classifier as commonly used in probability-of-default estimation [14]. This second stage probabilistic classifier $P_{2}^{p d}$ is trained to separate the defaulters from the non-defaulters; that is, the labeled dataset $\left\{\left(X_{i}^{k}, y_{i}\right)\right\}$.

The overall probability-of-default estimator with a reject option is then the following:

$$
p_{\text {sup_pd }}(X)= \begin{cases}-1 & \text { if } P_{1}^{\text {sup }}(y=+\mid X) \geq 0.5 \\ P_{2}^{p d}(y=+\mid X) & \text { if } P_{1}^{\text {sup }}(y=+\mid X)<0.5\end{cases}
$$

We note that it (wrongly) assumes that there are representative samples of all novel instances in the 'unknown' dataset. It estimates the likelihood of a client being accepted/rejected by the company, rather than how similar the instances are to the ones used to train the second stage classifier. It is hence no better equipped to detect completely novel instances than a single stage classifier.

\section{Background Check method}

As a third method, we use the Background Check technique proposed by Perello-Nieto e.a. [22]. This is an integrated methodology based on an explicit background class $b$, that they use to update the posterior distribution of the classifier as well as to detect ambiguous and novel cases.

Note that the method is developed in a multi-class setting where there can be more than two (foreground) classes $f_{c}$, but we apply it in a binary classification context. The goal of the method is to estimate for an instance $X$ the probability that $X$ belongs to the background class $P(b \mid X)$, that it belongs to the defaulters (here indicated by $\left.f_{1}\right) P\left(f_{1} \mid X\right)$ or to the non-defaulters $P\left(f_{0} \mid X\right)$. In order to calculate these probabilities, the authors introduce a familiarity factor $r(x)=$ $P(f \mid X) / P(b \mid X)$, i.e. the ratio of the posterior foreground probability to the posterior background probability, which, they show, also equals the ratio of the relative foreground density to relative background density $q_{f}(X) / q_{b}(X)$. They then compute the posterior probabilities using the following expressions:

$$
\begin{gathered}
P(b \mid X)=\frac{1}{1+r(X)} \\
P\left(f_{c} \mid X\right)=\frac{p\left(f_{c} \mid f, X\right) r(X)}{1+r(X)},
\end{gathered}
$$

i.e. by determining (i) the class posterior probabilities within foreground $p\left(f_{0} \mid f, X\right)$ and $p\left(f_{1} \mid f, X\right)$ and (ii) the familiarity factor $r(X)=q_{f}(X) / q_{b}(X)$.

Here, (i) can be found by using a standard multi-class probability estimator on the foreground data; that is, the known instances. The relative foreground density $q_{f}(X)$ in (ii) can be estimated using a standard density estimation algorithm on the foreground data, that is, an unsupervised learning method trained on all known data (in case of one-class SVM, with a sigmoid on top so that it ranges between 0 and 1 ).

Computing the relative background density $q_{b}(X)$ in (ii) is the hardest part as there is very little we know about it. We follow Perello-Nieto e.a. in assuming a background bias that takes the form of $q_{b}$ as an affine function of $q_{f}: q_{b}(X)=$ $\left(1-q_{f}(X)\right) \mu(0)+q_{f}(X) \mu(1)$, with $\mu(0)$ and $\mu(1)$ the socalled endpoints. In this work, we instantiate $\mu(0)$ and $\mu(1)$ so as to detect novel instances; that is, we assume that outliers lie in regions where the foreground data are less dense and thus expect the relative background and foreground densities to be each other's inverses. We therefore set $\mu(0)=1$ and $\mu(1)=0$, resulting in the affine transformation $q_{b}(x)=1-q_{f}(x)$. 
The resulting probability-of-default estimator with a reject option is then the following, assuming $P(b \mid X)$ and $P(f \mid X)$ are as defined in Eq. (9) and (10) respectively:

$$
p_{b g c}(X)= \begin{cases}-1 & \text { if } P(b \mid X) \geq \max _{c}\left(P\left(f_{c} \mid X\right)\right) \\ P\left(f_{1} \mid X\right) & \text { if } P(b \mid X)<\max _{c}\left(P\left(f_{c} \mid X\right)\right)\end{cases}
$$

We note that, compared to the two-stage method with unsupervised novelty detection, Background Check does not use a fixed threshold on the novelty score, but rather compares it to the maximum of the updated posterior distribution.

\section{Selection with Guaranteed Risk Control}

Finally, we also include a modern classification with reject option method, namely the SGR method proposed by Geifman and El-Yaniv [7]. The method is designed for the ambiguity reject setting, e.g. deciding when an instance is ambiguous and rejecting those. We include it to make the difference with our setting clear, as well as to empirically evaluate its assumptions on our datasets.

Aimed at ambiguity reject, they use as 'novelty' score $s(X)$ a confidence-rate function $\kappa$, more specifically the softmax response $\max _{c}\left(P^{n}(y=c \mid X)\right)$ for a given (neural) classifier $n$. In our binary classification setting, that means the closer an instance is to the decision boundary, the more likely it will be rejected. The basic assumption is that coverage (the number of instances selected) decreases as one moves away from the decision boundary, while risk (how difficult it is to separate them) decreases or conversely accuracy increases. They propose a well-found risk measure and a way to search for the optimal threshold $\theta^{*}$ that guarantees that risk within a certain confidence level $\delta$ [7].

Hence, the probability of default estimator with a reject option is the following:

$p_{s g r}(X)= \begin{cases}-1 & \text { if } \max _{c}\left(P^{n}(y=c \mid X)\right)<\theta^{*} \\ P^{n}(y=+\mid X) & \text { if } \max _{c}\left(P^{n}(y=c \mid X)\right) \geq \theta^{*}\end{cases}$

\section{REJECT INFERENCE WITH A CLASSIFIER WITH REJECT OPTION}

The approaches discussed so far, and classification with a reject option in general, are unrelated to reject inference. In reject inference, the main question is whether information from the unknown instances (called rejected instances in that community) can improve classification test accuracy on the known instances.

A common approach is to do semi-supervised learning, where iteratively part of the unknowns are labeled using a classifier trained on the labeled instances of the previous iteration. The idea behind such semi-supervised learning methods is to use the density of the unknown instances to bias the decision boundary [38].

In our setting we oppose the idea that all unknown instances can reliably be labeled. However, the purpose of the above approaches is exactly to identify which instances can reliably be labeled. We hence wish to evaluate whether reject inference on the unknown instances that get a reliable prediction can improve classification accuracy on the known instances.

This is a 2-step approach that assumes a standard probability of default estimator $P^{p d}$ and a probability of default estimator with reject option $p^{p d r}$. The training procedure is the following:

$$
\begin{aligned}
& \text { train } p^{p d r} \text { using }\left\{\left(X_{i}^{k}, y_{i}\right)\right\} \text { and }\left\{\left(X_{i}^{u}\right)\right\} \\
& \qquad y^{\prime}\left(X_{i}\right)= \begin{cases}\text { reject } & \text { if } p^{p d r}\left(X_{i}\right)=-1 \\
+ & \text { if } p^{p d r}\left(X_{i}\right) \geq 0.5 \\
- & \text { if } 0.5>p^{p d r}\left(X_{i}\right) \geq 0\end{cases} \\
& \text { let } D^{\prime}=\left\{\left(X_{i}^{k}, y_{i}\right)\right\} \cup\left\{\left(X_{i}^{u}, y^{\prime}\left(X_{i}\right)\right) \mid p^{p d r}\left(X_{i}\right) \neq-1\right\} \\
& \text { train } P^{p d} \text { on } D^{\prime}
\end{aligned}
$$

We hence do an iteration of semi-supervised learning and obtain a standard classifier trained on partially augmented data. We will experimentally verify the effectiveness of this in the next section.

\section{EXPERIMENTS}

\section{A. Datasets}

To investigate the performance of the previously introduced methods, we apply them to one artificial and six real datasets. Their target variables are all three-valued: either they consist of binary 'known' values and a quantifier meaning 'unknown' or they consist of three values and we choose one appropriate value to be the 'unknown' one as explained below. The dimensions and imbalance of each dataset are shown in Table II.

The artificial data was generated taking inspiration from the 2D PCA visualization of Fig. 1. The positive and negative instances are sampled from two somewhat overlapping Gaussians with the same variance for each, while the unknowns are sampled from a mixture of three Gaussians: one with high variance that overlaps both positives and negatives, one overlapping with the positives only and one non-overlapping that has low variance (see Fig. 2).

The first four real datasets are from OpenML [36], an open science platform for machine learning that enables sharing and organizing of data and therefore the reproducibility of experiments. They were selected among datasets with three labels based on the description of the labels and the suitability of treating one as 'unknown'. OpenML 26 Nursery contains data used to rank applications for nursery schools in Ljubljana [21]. The target variable's values 'priority' and 'special priority', i.e. the labels representing cases that are the most likely to be accepted, are resp. mapped to good and bad while the less desirable, possibly rejected rest is considered unknown. OpenML 1523 Vertebra Column contains values for six biomechanical features that are used to classify orthopaedic patients into three categories [24]. The target variable offers a natural dichotomy between 'normal' vertebrae on the one hand and 'spondylolisthesis' and 'disk hernia' cases on the other hand. These values are conveniently mapped to unknown resp. and good and bad resp. OpenML 4541 Diabetes 130US contains patient and hospital outcome data from 130 US 
TABLE II: Overview of datasets used.

\begin{tabular}{|c|c|c|c|c|}
\hline \multirow[t]{2}{*}{ Dataset } & \multirow{2}{*}{\multicolumn{2}{|c|}{$\begin{array}{c}\text { Dimensions } \\
\text { \# samples \# features }\end{array}$}} & \multicolumn{2}{|c|}{ Imbalance of target variable } \\
\hline & & & $\%$ good & \%unknown \\
\hline Artificial & 260 & 2 & \begin{tabular}{|lllll}
38.46 & $\% 8.46 \%$
\end{tabular} & $\% 23.08 \%$ \\
\hline 26 Nursery & 12,960 & 10 & $32.92 \% 31.20 \%$ & $\% 35.88 \%$ \\
\hline 1523 Vertebra Column & 310 & 8 & $48.39 \% \quad 19.35 \%$ & $\% 32.26 \%$ \\
\hline 4541 Diabetes 130US & 101,766 & 51 & $34.93 \% 11.16 \%$ & $\% 53.91 \%$ \\
\hline 1226 Click Prediction & 798,964 & 14 & $41.60 \% 8.40$ & $\% 50.00 \%$ \\
\hline Lending Club & $29,028,343$ & 10 & $\% 1.04$ & $\% 95.25 \%$ \\
\hline Invoice factoring & 6,773 & 119 & $73.88 \% 7.74$ & $\% 18.38 \%$ \\
\hline
\end{tabular}

hospitals and integrated delivery networks between 1999 and 2008 [30]. As a target variable, we use the variable specifying the days to inpatient readmission. We choose the value 'no record of readmission' to be the unknown value, since we don't know if patients were readmitted in another hospital. We map the values ' $<30$ days' and ' $>30$ days' to bad and good respectively. OpenML 1226 Click Prediction was obtained from the $2012 \mathrm{KDD}$ Cup. It contains search engine query and user info. The target variable's values indicate whether or not a user clicked on at least one ad alongside the search engine results and are mapped to resp. bad and good. The rest of the values are missing and therefore treated as unknown.

The last two datasets concern the credit risk domain. One dataset contains loan data issued between 2017 and 2018 from Lending Club, a P2P lending service which is made available to borrowers. The data shows a high rejection rate as can be expected in a P2P lending context. As a target variable, we take the loan status of fully paid (good) loans or defaulted or late $(\mathrm{bad})$ loans. The rejected instances are then unknown. The final dataset is a private dataset over a period of two years at a European spot factoring company; that is, credit lending for individual invoices. Besides a variable indicating whether or not an invoice's outcome was good or bad, the dataset contains rejected (unknown) instances as well.

\section{B. Experiment setup}

Unless specified otherwise, we use classifiers from the scikit-learn (v0.22.2) machine learning library. As standard probability-of-default classifier $P^{p d}$ we use the RandomForestClassifier. We will compare two variants of $p_{\text {unsup_pd }}$ : one that uses IsolationForest as unsupervised learning method (unsup_pd_if) and one using OneClassSVM (unsup_pd_oc). $p_{\text {sup_pd }}$ uses a random forest for both stages. The implementations for SGR and Background Check are from the GitHub repositories of their respective authors.

All methods are evaluated using 5-fold outer crossvalidation and 3-fold inner cross-validation. This includes hyperparameter optimization across the most common hyperparameters for the methods considered, using the product of the coverage and unknown-is-positive accuracy as evaluation measure. SGR additionally requires a risk bound, which we set to 0.3 as lower values often lead to solely reject predictions on the binary classification data. Due to their sizes and the effort of this grid search, the LendingClub and Click dataset were downsampled in a stratified way to $10 \%$ and $50 \%$ respectively.
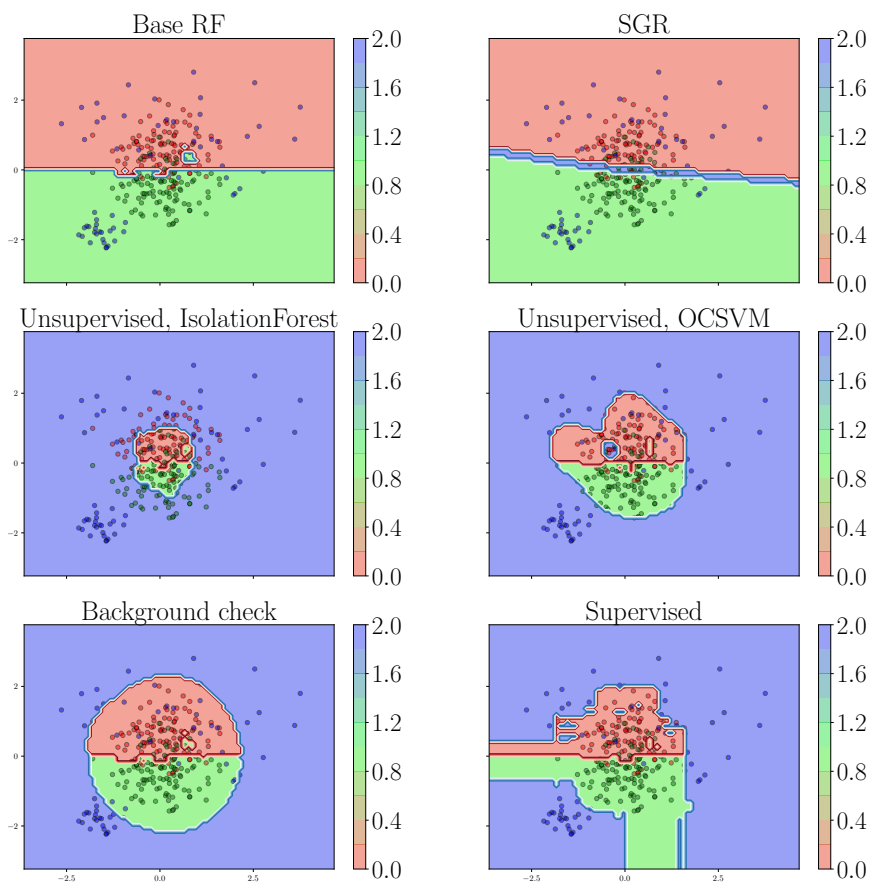

Fig. 2: The decision boundary of all methods applied to an artificial dataset. Blue indicates rejected instances, red indicates positive predictions and green negative predictions.

\section{Experiment 1: Visualizations of toy data predictions}

We start with a visualization of the classification boundaries on our 2D artificial data, see Fig. 2. Such a visual inspection already highlights important differences between the different methods. We can observe that Base RF never rejects an instance and has decision boundaries and prediction areas that continue indefinitely. SGR is devised for the ambiguity reject and we can see that it rejects instances around the decision boundary, unrelated to the unknowns. The other four methods successfully bound the region in which they perform classification around the observed instances, but differ in exact regions used. Using isolation forests leads to the most conservative results, with many known instances in the reject category. The decision space associated with the one-class SVM (OCSVM) shows remarkably well defined regions. Background Check also uses one-class SVMs, albeit to estimate the class probabilities of the unknown vs. known classes, rather than as a preliminary outlier detection. This seems to lead to more regular decision areas that are less desirable for this toy data. Finally, supervised learning also produces well-separated classifications, but only for the regions where unknowns are present. In regions where no unknowns are observed, the decision boundary and prediction area of the second stage classifier also continues indefinitely and an arbitrary prediction is made.

\section{Experiment 2: Quantitative comparison}

In the second experiment we apply each method to the artificial and six real datasets, compute the extended confusion 
TABLE III: Comparison across the real datasets.

\begin{tabular}{|c|c|c|c|c|c|c|}
\hline 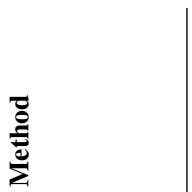 & 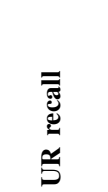 & 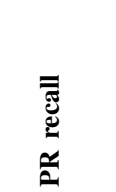 & $\begin{array}{l}\overline{\bar{J}} \\
\text { 巳一 } \\
\text { Z }\end{array}$ & 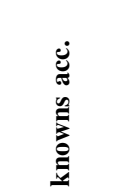 & 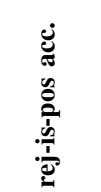 & 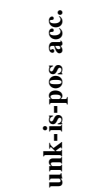 \\
\hline sgr & $88.33 \%$ & $95.00 \%$ & $98.00 \%$ & $28.57 \%$ & $48.50 \%$ & $14.29 \%$ \\
\hline 5 unsu & $96.67 \%$ & $58.00 \%$ & $68.00 \%$ & $6.22 \%$ & $53.50 \%$ & $67.11 \%$ \\
\hline uns & $76.67 \%$ & $84.00 \%$ & $83.00 \%$ & $100.00 \%$ & $58.50 \%$ & $89.36 \%$ \\
\hline bgch & $61.67 \%$ & $1.00 \%$ & $0.00 \%$ & $80.40 \%$ & $80.50 \%$ & $79.28 \%$ \\
\hline sup_pd & $65.00 \%$ & $3.00 \%$ & $1.00 \%$ & $77.04 \%$ & $77.00 \%$ & $76.04 \%$ \\
\hline $\operatorname{sgr}$ & $0.00 \%$ & $0.00 \%$ & $0.00 \%$ & $98.57 \%$ & $98.57 \%$ & $72.36 \%$ \\
\hline unsu & $100.00 \%$ & $100.00 \%$ & $100.00 \%$ & $\mathrm{NaN}$ & $48.66 \%$ & $\mathrm{NaN}$ \\
\hline unsu & $94.17 \%$ & $64.49 \%$ & $44.23 \%$ & $99.29 \%$ & $76.97 \%$ & $92.90 \%$ \\
\hline bgch & $43.63 \%$ & $0.00 \%$ & $0.02 \%$ & $99.41 \%$ & $99.40 \%$ & $82.66 \%$ \\
\hline$\stackrel{\sim}{\sim}$ sup_l & $98.06 \%$ & $0.00 \%$ & $0.00 \%$ & $99.21 \%$ & $99.21 \%$ & $98.14 \%$ \\
\hline sgr & $80.00 \%$ & 80.0 & $\overline{80}$ & $8 \%$ & $40.95 \%$ & $67.74 \%$ \\
\hline uns & $30.00 \%$ & $26.67 \%$ & $.67 \%$ & $18 \%$ & $73.33 \%$ & $91.98 \%$ \\
\hline I_oc & $46.00 \%$ & $18.33 \%$ & $56.00 \%$ & & $57.62 \%$ & $94.67 \%$ \\
\hline bgche & $0.00 \%$ & $0.00 \%$ & $0.67 \%$ & $98.09 \%$ & $97.62 \%$ & $93.85 \%$ \\
\hline sup_pd & $70.00 \%$ & $28.33 \%$ & $2.67 \%$ & $99.47 \%$ & $97.62 \%$ & $95.89 \%$ \\
\hline S & $99.93 \%$ & $99.95 \%$ & 99 & $6 \%$ & $24.26 \%$ & $39.19 \%$ \\
\hline$x^{2}$ & $0.43 \%$ & 0. & $\%$ & $9 \%$ & $76.09 \%$ & $35.89 \%$ \\
\hline d_oc & $73.43 \%$ & $63.69 \%$ & $65.45 \%$ & $4 \%$ & $1.77 \%$ & $41.30 \%$ \\
\hline$\overline{7} \mathrm{bgc}$ & $0.02 \%$ & $0.05 \%$ & $0.06 \%$ & $76.38 \%$ & $76.35 \%$ & $36.20 \%$ \\
\hline$\approx$ sup_l & $70.44 \%$ & $40.49 \%$ & $39.77 \%$ & $76.29 \%$ & $55.62 \%$ & $49.11 \%$ \\
\hline 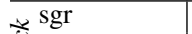 & $00 \%$ & $0.00 \%$ & $0.00 \%$ & $21 \%$ & $83.21 \%$ & $41.60 \%$ \\
\hline & $59 \%$ & $7.29 \%$ & $43 \%$ & $76 \%$ & $9.48 \%$ & $42.36 \%$ \\
\hline d_oc & $99.47 \%$ & $99.65 \%$ & $99.46 \%$ & $.33 \%$ & $17.19 \%$ & $46.80 \%$ \\
\hline bochel. & $0.01 \%$ & $0.06 \%$ & $0.00 \%$ & $83.15 \%$ & $83.16 \%$ & $46.42 \%$ \\
\hline- sup_pd & $42.09 \%$ & $46.93 \%$ & $45.52 \%$ & $84.03 \%$ & $53.46 \%$ & $41.22 \%$ \\
\hline & $100.00 \%$ & $100.00 \%$ & $100.00 \%$ & $\mathrm{NaN}$ & $21.95 \%$ & $0.00 \%$ \\
\hline pd_if & $56.33 \%$ & $23 \%$ & $\%$ & $6.95 \%$ & $68.12 \%$ & $14.95 \%$ \\
\hline d_oc & $99.68 \%$ & 42.9 & $40.10 \%$ & $8.85 \%$ & $56.16 \%$ & $71.25 \%$ \\
\hline$\cong$ bgcheck & $56.41 \%$ & $0.00 \%$ & $0.01 \%$ & $76.93 \%$ & $76.93 \%$ & $18.78 \%$ \\
\hline ปั sup_pd & $100.00 \%$ & $0.00 \%$ & $0.00 \%$ & $78.03 \%$ & $78.03 \%$ & $78.03 \%$ \\
\hline sgr & $41.69 \%$ & $47.14 \%$ & $42.65 \%$ & $0.47 \%$ & $55.97 \%$ & $74.85 \%$ \\
\hline$\cong$ uns & & & & & $90.67 \%$ & $74.66 \%$ \\
\hline$=$ & $51.97 \%$ & $23.09 \%$ & $22.48 \%$ & $3 \%$ & $72.77 \%$ & $80.00 \%$ \\
\hline & $3.29 \%$ & $0.57 \%$ & $0.44 \%$ & $91.66 \%$ & $91.30 \%$ & $75.46 \%$ \\
\hline sup_p & $78.71 \%$ & $2.29 \%$ & $1.42 \%$ & $91.09 \%$ & $89.94 \%$ & $86.95 \%$ \\
\hline
\end{tabular}

matrices introduced in Section IV and the derived metrics. The results can be seen in Table III. The first three columns show the recall of rejects. A high unknown reject recall corresponds to a high amount of rejected unknowns, but also a low amount of 'missed opportunities' retrieved from the unknown population. The positive and negative reject recall represent knowns that are now rejected and therefore they better have a low value. The 'knowns accuracy' shows the accuracy of the classifier in terms of the original definition of accuracy and it should therefore be high. The last two columns concern derived accuracy metrics as explained in Section IV.

The results vary from dataset to dataset. Regarding the artificial data, we can see that one-class SVM performs best with a remarkable $100 \%$ classification accuracy, though most of the positives and negatives are rejected. For the Nursery dataset, isolation forest rejects all while SGR rejects none, both of which is undesirable. Background Check and the supervised approach show high known accuracies, low known reject recall rates and elevated unknown reject recall rates as desired.

In the Vertebra dataset, Background Check rejects almost none while still obtaining the second highest accuracy. The supervised method is here best at rejecting more unknowns than knowns. In Diabetes, SGR rejects almost all samples while achieving very high accuracy on the knowns but with bad results when taking unknowns into account. Except for the supervised method, all methods seem to reject a similar proportion of instances in the knowns and unknowns. All methods show low unknown-is-pos accuracy values here. The Click data proves difficult for all methods.

As for the first credit dataset, Lending Club, which contains more then $95 \%$ unknowns, we can see a good separation of unknowns for all but SGR. Notably, the supervised method can separate all instances. Background Check manages to rejects a lot of the unknowns and no known as well, even though it received no information on the unknowns.

In the Factoring credit dataset, both the unsupervised method with isolation forest and the Background Check method reject almost no samples, surprisingly, but have high knowns accuracy values. The supervised method scores high on knowns accuracy as well, but manages to reject a lot of the unknowns while rejecting almost no unknown.

In general, the SGR method often has the same reject recall for knowns and unknowns, highlighting its mismatch with the goal. The supervised approach often performs best, but we must remark that it may make unreliable predictions on the unknowns that we can not evaluate here. Background Check shows similar good accuracy values but has more difficulties with respect to separating the unknowns. The twostage unsupervised methods also perform reasonably well and similar despite the visual differences in the previous experiment. Under the assumption of 'reject is positive' the accuracy of the isolation forest method is typically higher than OCSVM, except for the artificial and Nursery dataset.

\section{E. Experiment 3: Accuracy-rejection curves}

In the previous experiment, the methods were expected to use a hard threshold to determine whether an instance is novel or not. In the following experiment we will use accuracyrejection plots, with accuracy computed on the knowns only, to investigate the importance of the novelty thresholds.

Fig. 3 shows the results on the two credit risk datasets. We can see that for rejection rates higher than $90 \%$, the curves become unstable which is a side effect of evaluating over increasingly smaller sets of instances.

We can see that SGR performs well in these plots, albeit because this plot favours ambiguity rejects. It should however be noted that while the other methods focus on identifying novel instances rather than ambiguous instances, they achieve similar behavior in accuracies for increasing rejection thresholds.

\section{F. Experiment 4: Semi-supervised learning}

In this experiment, we investigate the benefit of novelty reject for reject inference. We use the 2-step approach discussed in Section VI, where the training data is augmented with nonrejected labels assigned by the proposed methods. A standard random forest is then trained on this augmented data. 

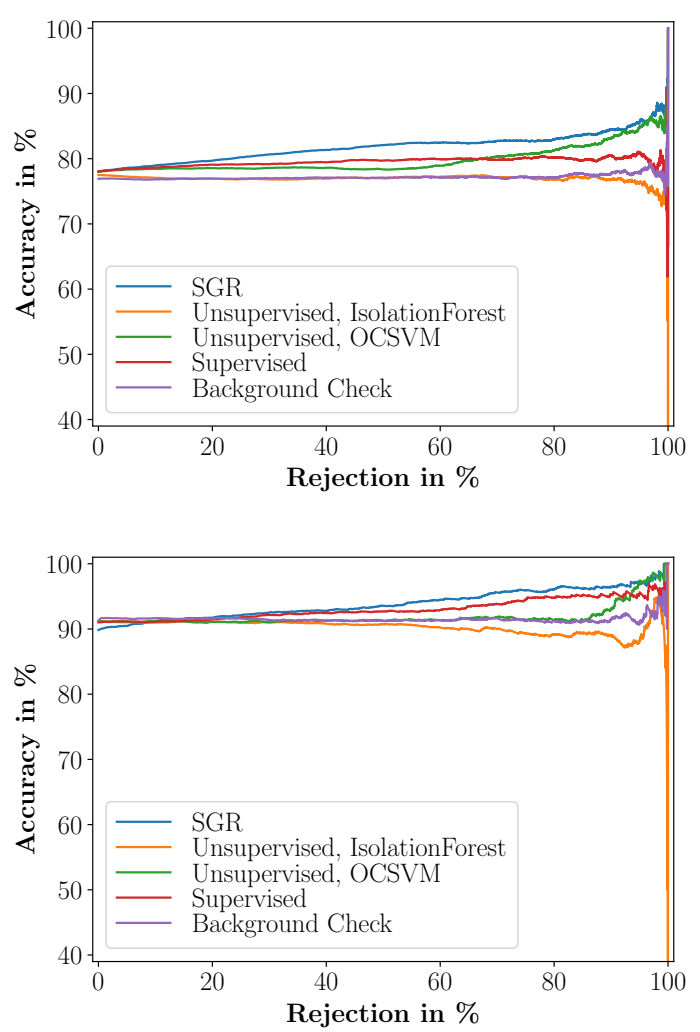

Fig. 3: AR curves for Lending Club (upper plot) and Factoring dataset (lower plot).

Table IV depicts the results of the experiment. We use the standard supervised learning metrics AUC, accuracy and f1measure to compare the results without the semi-supervised step (Base RF) to those with semi-supervised augmentation. The best and second best values per dataset and metric are resp. shown in bold and italic.

A first point worth mentioning is that the AUC and accuracy values are pretty similar across different methods. Indeed, few unknown instances will typically get a label and the additional information is expected to not induce abrupt changes in the predictions but rather local shifts in the decision boundaries.

Compared to the other methods, Base RF has slightly higher values for AUC for all datasets except for the Nursery and Diabetes datasets, where resp. the Background Check and supervised methods show slightly better AUC values. Base RF and Background Check also score well on the accuracy and f1-measure: in all datasets except Click, one or both of these methods can be found as best or second best performer.

We can observe good results for the supervised and unsupervised methods as well in several datasets.

\section{DISCUSSION \& CONCLUSION}

We motivated the problem of probability-of-default estimation with a reject option, motivated by credit scoring applications. In this domain the 'through-the-door population' is typically much wider than the 'accepted' customers on
TABLE IV: Results of the fourth experiment.

\begin{tabular}{|c|c|c|c|c|}
\hline 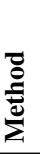 & & 气 & 导 & 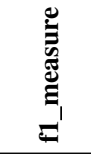 \\
\hline \multirow{6}{*}{ 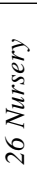 } & Base RF & $99.98 \%$ & $99.25 \%$ & $99.27 \%$ \\
\hline & $\operatorname{sgr}$ & $99.93 \%$ & $98.57 \%$ & $98.60 \%$ \\
\hline & unsup_pd_if & $99.95 \%$ & $98.81 \%$ & $98.83 \%$ \\
\hline & unsup_pd_oc & $99.95 \%$ & $98.69 \%$ & $98.71 \%$ \\
\hline & bgcheck & $99.99 \%$ & $99.31 \%$ & $99.33 \%$ \\
\hline & sup_pd & $99.95 \%$ & $98.69 \%$ & $98.71 \%$ \\
\hline \multirow{6}{*}{ 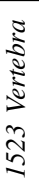 } & Base RF & $99.89 \%$ & $98.10 \%$ & $98.67 \%$ \\
\hline & sgr & $99.81 \%$ & $98.10 \%$ & $98.67 \%$ \\
\hline & unsup_pd_if & $99.45 \%$ & $98.10 \%$ & $98.67 \%$ \\
\hline & unsup_pd_oc & $99.76 \%$ & $98.10 \%$ & $98.67 \%$ \\
\hline & bgcheck & $99.64 \%$ & $98.10 \%$ & $98.67 \%$ \\
\hline & sup_pd & $99.83 \%$ & $98.57 \%$ & $99.00 \%$ \\
\hline \multirow{6}{*}{ 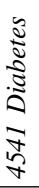 } & Base RF & $61.65 \%$ & $76.34 \%$ & $86.32 \%$ \\
\hline & sgr & $58.87 \%$ & $75.93 \%$ & $86.27 \%$ \\
\hline & unsup_pd_if & $59.05 \%$ & $75.94 \%$ & $86.27 \%$ \\
\hline & unsup_pd_oc & $60.68 \%$ & $76.18 \%$ & $86.33 \%$ \\
\hline & bgcheck & $59.73 \%$ & $76.20 \%$ & $86.38 \%$ \\
\hline & sup_pd & $61.68 \%$ & $76.20 \%$ & $86.30 \%$ \\
\hline \multirow{6}{*}{ 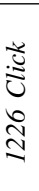 } & Base RF & $69.40 \%$ & $83.12 \%$ & $90.53 \%$ \\
\hline & $\operatorname{sgr}$ & $65.59 \%$ & $83.36 \%$ & $90.86 \%$ \\
\hline & unsup_pd_if & $65.48 \%$ & $\mathbf{8 3 . 5 2} \%$ & $90.92 \%$ \\
\hline & unsup_pd_oc & $67.54 \%$ & $83.23 \%$ & $90.62 \%$ \\
\hline & bgcheck & $66.48 \%$ & $83.20 \%$ & $90.59 \%$ \\
\hline & sup_pd & $66.83 \%$ & $83.46 \%$ & $90.82 \%$ \\
\hline \multirow{6}{*}{ 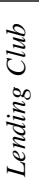 } & Base RF & $59.68 \%$ & $77.72 \%$ & $\mathbf{8 7 . 4 1} \%$ \\
\hline & $\operatorname{sgr}$ & $57.88 \%$ & $77.00 \%$ & $86.87 \%$ \\
\hline & unsup_pd_if & $57.87 \%$ & $77.06 \%$ & $86.90 \%$ \\
\hline & unsup_pd_oc & $58.06 \%$ & $76.89 \%$ & $86.78 \%$ \\
\hline & bgcheck & $57.69 \%$ & $76.92 \%$ & $86.83 \%$ \\
\hline & sup_pd & $57.85 \%$ & $76.89 \%$ & $86.78 \%$ \\
\hline \multirow{6}{*}{ 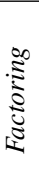 } & Base RF & $\mathbf{8 0 . 5 2 \%}$ & $91.41 \%$ & $95.44 \%$ \\
\hline & sgr & $78.10 \%$ & $90.81 \%$ & $95.13 \%$ \\
\hline & unsup_pd_if & $78.06 \%$ & $90.96 \%$ & $95.21 \%$ \\
\hline & unsup_pd_oc & $78.36 \%$ & $91.05 \%$ & $95.25 \%$ \\
\hline & bgcheck & $79.53 \%$ & $\mathbf{9 1 . 5 3} \%$ & $95.49 \%$ \\
\hline & sup_pd & $78.90 \%$ & $91.01 \%$ & $95.22 \%$ \\
\hline
\end{tabular}

which we can train predictive models. We identified the task of only providing estimates for clients that are similar to the customers with an observed outcome. It is hence a combination of novelty detection and probability-of-default estimation.

Out of four proposed methods, using a supervised method to separate the unknowns from the knowns worked surprisingly well, even though it can not inherently identify truly novel cases. The Background Check method matched the task really well and had high accuracy values, but had more difficulties than the supervised method in separating the unknowns from the knowns within its rejects, as expected. Simpler two-stage methods that used an unsupervised method and did not modify posterior probabilities performed good as well, but often did not make a difference between knowns and unknowns when rejecting.

Interestingly the accuracy-rejection curves showed that similar slight accuracy increases are possible for novelty reject methods as well as the ambiguity reject method. This raises the question of whether it would be valuable to do both ambiguity and novelty rejects in a single method. Another key direction 
of research is to further exploit the given 'unknown' data: even though their label is unknown, their presence could be used to better determine the novelty score threshold to use.

In general, many more techniques and combinations for the novelty-plus-probability-of-default combination can be devised. However, a key difficulty is evaluating the effect of the method on the unknowns. We proposed a number of metrics, but ideally one could evaluate in real life by following the recommendations of the system blindly; which few financial institutions are willing to do.

\section{ACKNOWLEDGMENT}

This project was funded by the Institute for the encouragement of Scientific Research \& Innovation of Brussels (Innoviris, 2017-COTEAM-UP-19).

\section{REFERENCES}

[1] Baesens, B., Rösch, D., Scheule, H.: Credit risk analytics. John Wiley \& Sons, Inc. (2016)

[2] Bishop, C.M.: Pattern Recognition and Machine Learning. Springer (2006)

[3] Chen, G.G., Astebro, T.: The Economic Value of Reject Inference in Credit Scoring. In: L. C. Thomas, J. N. Crook and D. B. Edelman (eds.): Credit Scoring and Credit Control VII, Proceedings of Conference held at University of Edinburgh (2001)

[4] Chow, C.: On optimum recognition error and reject tradeoff. IEEE Transactions on Information Theory 16(1), 41-46 (1970)

[5] DeVries, T., Taylor, G.W.: Learning Confidence for Out-of-Distribution Detection in Neural Networks. arXiv preprint arXiv:1802.04865 (2018)

[6] Dubuisson, B., Masson, M.: A statistical decision rule with incomplete knowledge about classes. Pattern Recognition 26(1), 155-165 (1993)

[7] Geifman, Y., El-Yaniv, R.: Selective Classification for Deep Neura Networks. In: Guyon, I., Luxburg, U.V., Bengio, S., Wallach, H., Fergus, R., Vishwanathan, S., Garnett, R. (eds.) Advances in Neural Information Processing Systems 30, pp. 4878-4887. Curran Associates, Inc. (2017)

[8] Guan, H., Zhang, Y., Cheng, H., Xian, M., Tang, X.: BA2Cs: Bounded abstaining with two constraints of reject rates in binary classification. Neurocomputing 357, 125-134 (2019)

[9] Hand, D., Henley, W.: Can reject inference ever work? IMA Journal of Management Mathematics 5(1), 45-55 (1993)

[10] Heckman, J.J.: The Common Structure of Statistical Models of Truncation, Sample Selection and Limited Dependent Variables and a Simple Estimator for Such Models. Annals of Economic and Social Measurement 5(4), 475-492 (1976)

[11] Hendrycks, D., Gimpel, K.: A Baseline for Detecting Misclassified and Out-of-Distribution Examples in Neural Networks. International Conference on Learning Representations (ICLR) (2017)

[12] Kozodoi, N., Katsas, P., Lessmann, S., Moreira-Matias, L., Papakonstantinou, K.: Shallow Self-Learning for Reject Inference in Credit Scoring. In: Brefeld U., Fromont E., Hotho A., Knobbe A., Maathuis M., Robardet C. (eds) Machine Learning and Knowledge Discovery in Databases. ECML PKDD 2019. Lecture Notes in Computer Science, vol 11908. Springer, Cham (2020)

[13] Lee, K., Lee, H., Lee, K., Shin, J.: Training Confidence-calibrated Classifiers for Detecting Out-of-Distribution Samples. ICLR 2018 (2018)

[14] Lessmann, S., Baesens, B., Seow, H.V., Thomas, L.C.: Benchmarking state-of-the-art classification algorithms for credit scoring: An update of research. European Journal of Operational Research 247(1), 124-136 (2015)

[15] Liang, S., Li, Y., Srikant, R.: Enhancing The Reliability of Out-ofdistribution Image Detection in Neural Networks. ICLR 2018 (2018)

[16] Liu, F.T., Ting, K.M., Zhou, Z.H.: Isolation Forest. In: 2008 Eighth IEEE International Conference on Data Mining. pp. 413-422. IEEE, Pisa, Italy (2008)
[17] Maldonado, S., Paredes, G.: A Semi-supervised Approach for Reject Inference in Credit Scoring Using SVMs. In: Hutchison, D., Kanade, T., Kittler, J., Kleinberg, J.M., Mattern, F., Mitchell, J.C., Naor, M., Nierstrasz, O., Pandu Rangan, C., Steffen, B., Sudan, M., Terzopoulos, D., Tygar, D., Vardi, M.Y., Weikum, G., Perner, P. (eds.) Advances in Data Mining. Applications and Theoretical Aspects, vol. 6171, pp. 558571. Springer Berlin Heidelberg, Berlin, Heidelberg (2010)

[18] Masana, M., Ruiz, I., Serrat, J., van de Weijer, J., Lopez, A.M.: Metric Learning for Novelty and Anomaly Detection. In Proceedings of the British Machine Vision Conference (2018)

[19] Mohseni, S., Pitale, M., Yadawa, J., Wang, Z.: Self-Supervised Learning for Generalizable Out-of-Distribution Detection. AAAI 2020 pp. 5216$5223(2020)$

[20] Nadeem, M.S.A., Zucker, J.D., Hanczar, B.: Accuracy-Rejection Curves (ARCs) for Comparing Classification Methods with a Reject Option. In Machine Learning in System Biology, Journal of Machine Learning, volume 8 of JMLR Proceedings pp. 65-81 (2010)

[21] Olave, M., Rajkovič, V., Bohanec, M.: An application for admission in public school systems. In: (I. Th. M. Snellen and W. B. H. J. van de Donk and J.-P. Baquiast, editors) Expert Systems in Public Administration, pp. 145-160. Elsevier Science Publishers (North Holland) (1989)

[22] Perello-Nieto, M., Filho, T.D.M.E.S., Kull, M., Flach, P.: Background Check: A General Technique to Build More Reliable and Versatile Classifiers. In: 2016 IEEE 16th International Conference on Data Mining (ICDM). pp. 1143-1148. IEEE, Barcelona, Spain (2016)

[23] Puhani, P.: The Heckman Correction for Sample Selection and Its Critique. Journal of Economic Surveys 14(1), 53-68 (2000)

[24] da Rocha Neto, A.R., Sousa, R., de A. Barreto, G., Cardoso, J.S.: Diagnostic of Pathology on the Vertebral Column with Embedded Reject Option. In: Vitrià, J., Sanches, J.M., Hernández, M. (eds.) Pattern Recognition and Image Analysis, vol. 6669, pp. 588-595. Springer Berlin Heidelberg (2011)

[25] Schultheiss, A., Käding, C., Freytag, A., Denzler, J.: Finding the Unknown: Novelty Detection with Extreme Value Signatures of Deep Neural Activations. In: Roth, V., Vetter, T. (eds.) Pattern Recognition, vol. 10496, pp. 226-238. Springer International Publishing, Cham (2017)

[26] Schölkopf, B., Platt, J.C., Shawe-Taylor, J., Smola, A.J., Williamson, R.C.: Estimating the Support of a High-Dimensional Distribution. Neural Computation 13(7), 1443-1471 (2001)

[27] Sheng, V.S., Ling, C.X.: Thresholding for Making Classifiers Costsensitive. AAAI '06 Proceedings of the 21st national conference on Artificial intelligence 1, 476-481 (2006)

[28] Siddiqi, N.: Credit risk scorecards: developing and implementing intelligent credit scoring. Wiley, Hoboken, N.J (2006)

[29] Stehman, S.V.: Selecting and interpreting measures of thematic classification accuracy. Remote Sensing of Environment 62(1), 77-89 (1997)

[30] Strack, B., DeShazo, J.P., Gennings, C., Olmo, J.L., Ventura, S., Cios, K.J., Clore, J.N.: Impact of HbA1c Measurement on Hospital Readmission Rates: Analysis of 70,000 Clinical Database Patient Records. BioMed Research International 2014, 1-11 (2014)

[31] Tax, D., Duin, R.: Uniform object generation for optimizing one-class classifiers. J. Mach. Learn. Res. pp. 155-173 (2001)

[32] Tax, D., Duin, R.: Growing a multi-class classifier with a reject option. Pattern Recognition Letters 29(10), 1565-1570 (2008)

[33] Thulasidasan, S., Bhattacharya, T., Bilmes, J., Chennupati, G., MohdYusof, J.: Combating Label Noise in Deep Learning Using Abstention. In International Conference on Machine Learning pp. 6234-6243 (2019)

[34] Thulasidasan, S., Bhattacharya, T., Bilmes, J., Chennupati, G., Mohd-Yusof, J.: Knows When it Doesn't Know: Deep Abstaining Classifiers. OpenReview.net (2018) https: / / openreview . net/forum?id=rJxF73R9tX

[35] Tian, Y., Yong, Z., Luo, J.: A new approach for reject inference in credit scoring using kernel-free fuzzy quadratic surface support vector machines. Applied Soft Computing 73, 96-105 (2018)

[36] Vanschoren, J., van Rijn, J.N., Bischl, B., Torgo, L.: OpenML: networked science in machine learning. ACM SIGKDD Explorations Newsletter 15(2), 49-60 (2014)

[37] Xia, Y.: A Novel Reject Inference Model Using Outlier Detection and Gradient Boosting Technique in Peer-to-Peer Lending. IEEE Access 7 , 92893-92907 (2019)

[38] Zhu, X.J.: Semi-Supervised Learning Literature Survey. Technical Report, University of Wisconsin-Madison Department of Computer Sciences (2005) 